\title{
Lenguas indígenas vivas del Ecuador
}

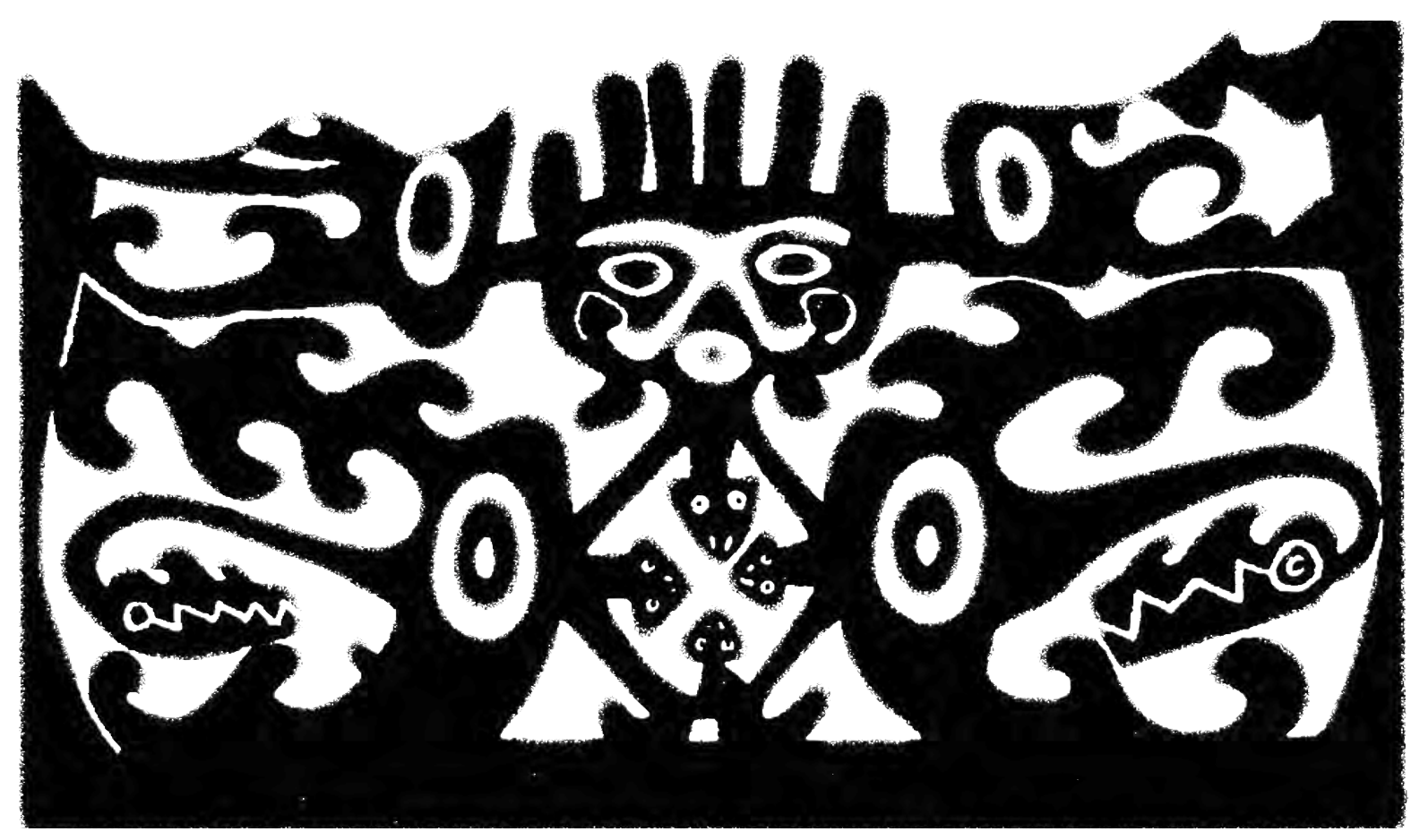

\section{Introducción}

El Ecuador no comenzó hace doscientos años con la pseudoindependencia de los terratenientes criollos y grupos de poder. El Ecuador comenzó hace más de quince mil años. Según varios estudios, en 1822 la población ecuatoriana estaba conformada de la siguiente manera: un setenta por ciento era indígena, un ocho por ciento era población afroecuatoriana, un diez por ciento era mestiza y el resto era criolla (españoles nacidos en el Ecuador) y chapetona (españoles nacido en España). Ese pequeño grupo de criollos se apropió del poder y del futuro del Ecuador. En toda América Latina, las guerras de la "independencia" fueron guerras entre españoles: los criollos terratenientes contra los chapetones que ocupaban los cargos di- 
rectivos de nuestros países. Fue una pelea por el poder entre marqueses y dignatarios y a población indígena, negra y mestiza fue utilizada por ambos bandos. La mayor parte de la población ecuatoriana fue marginada desde el inicio de la vida republicana. Para asegurar el dominio, este pequeño grupo de poder organizó los llamados "ejércitos nacionales" con un tinte patriotero y elitista plagado de privilegios.

La mayor parte de los libros de historia del Ecuador fueron escritos según la interpretación del pequeño grupo de criollos que se adueñó del poder a comienzos del siglo XIX. La imposición de esta visión parcializada de la historia a través de la educación, contribuyó a la formación de una diglosia en la sociedad ecuatoriana. ${ }^{3}$ Para superar esta situación, entre otras cosas, hay que conocer la importancia del estudio de las lenguas y culturas indígenas y su contribución al desarrollo sustentable del Ecuador.

El nombre que lleva Quito transmite el significado de una lengua milenaria aún viva. El sentido de esta palabra representa el gran avance científico de nuestros antepasados. To o Tu en lenguas tsa'fiki y cha'fiki respectivamente, significa tierra y Qui (de quitsa), significa mitad. Por lo tanto, Quito o Quitu quiere decir “Tierra en la mitad del Mundo". Las investigaciones lingüísticas, arqueológicas, históricas, etc., muestran que los pueblos tsachi y chachi, que ahora sólo están en la Costa, estuvieron también en la Sierra desde hace miles de años. El contacto entre los pueblos de la la Costa, Sierra y Amazonía parece que fue muy intenso antes de la invasión española.

Los avances científicos de las diferentes culturas existentes, antes de la invasión europea, fueron importantes en varios aspectos: agricultura, nutrición, salud, música, danza, astronomía, arquitectura, etc. Todo esto lo llevamos los ecuatorianos en nuestros genes. Por razones de espacio, este estudio se referirá fundamentalmente al estado de las investigaciones lingüísticas relacionadas con su uso en la educación intercultural bilingüe, y solo colateral- mente se referirá a otras contribuciones de las culturas indígenas al desarrollo sustentable del Ecuador.

El elemento más visible de una cultura es la lengua. En ella están expresados los conocimientos, creencias, actitudes, ciencia, tecnología y cosmovisión del pueblo que la ha forjado a lo largo de centurias o milenios. Se puede decir que cuando una lengua muere la cultura comienza su agonía.

Las nacionalidades indígenas del Ecuador han conseguido incluir en la Constitución Política de la República el reconocimiento de la educación intercultural bilingüe como uno de los derechos colectivos, fundamentada en las lenguas y culturas milenarias. Además, han generado un proceso educativo de crecimiento humano, fundamentado en la sabiduría milenaria de los pueblos indígenas y de otros pueblos del mundo a nivel universal.

El elemento más visible de una cultura es la lengua, por cuanto en ella están expresados los conocimientos, creencias, actitudes, ciencia, tecnología y cosmovisión del pueblo que la ha forjado a lo largo de centurias o milenios. Por ello se puede decir que cuando una lengua muere, la cultura no tarda en desaparecer en sus aspectos más vitales.

Son diversos los grados de avance de a las investigaciones sobre las once lenguas indígenas existentes en el país y de sus variaciones dialectales. Las que están en la Amazonía son: quichua, shuar-achuar-shiwiar (aents chicham), wao tededo, a'ingae, paikoka, zápara, y andoa. En la Sierra está presente el Quichua. En la Costa están: awapit, cha'fiki, tsa'fiki, y epera pedede. La mayor parte de los estudios de las lenguas indígenas realizadas son de carácter descriptivo 
y aislado respecto al proceso educativo de los pueblos indígenas.

Existen pocas investigaciones destinadas a apoyar el proceso de fortalecimiento de las lenguas y culturas del Ecuador. Las que más estudios tienen, aunque sea de tipo descriptivo, son la quichua y la shuar. De las demás existen pocos estudios y de algunas como el epera pedede apenas hay una primera aproximación a la fonología para el habla de la variante ecuatoriana.

Uno de los primeros pasos para escribir en lenguas indígenas y posibilitar que los hablantes las representen por escrito es contar con un sistema de escritura. El sistema de escritura debe contener un alfabeto (signos gráficos) y unas reglas ortográficas que permitan el funcionamiento del código escrito.

El proceso de establecimiento de la escritura de una lengua es complejo. La complejidad no se debe tanto al estudio lingüístico, sino al hecho de que la escritura es una construcción psicosocial en la que intervienen factores de poder político y religioso. Para establecer la escritura de una lengua debería tomarse en cuenta el estudio de la fonología y algunos aspectos relacionados con la facilidad de aprendizaje por parte de los niños. Es decir, se debería descubrir e inventariar los fonemas existentes en la lengua y a cada fonema asignarle un signo escrito (letra) para que lo represente. Pero en la realidad se mezclan otros factores. Por ejemplo, para varias lenguas indígenas el Instituto Lingüístico de Verano ILV, de la Universidad de Oklahoma, Estados Unidos, asignó signos y reglas parecidos al código del español. Tradujeron la Biblia a las lenguas indígenas y para escribirla siguieron el código del la escritura del español.

Por ejemplo, el problema de la $h$ muda, que en español tiene su historia, se trasladó a la lengua indígena. En español, hace varios siglos la $h$ sonaba, pero luego desapreció a nivel oral; sin embargo, en el escrito se la mantiene hasta hoy. En las lenguas indígenas, este problema es totalmente extraño. En las lenguas indígenas en lugar de hua, hue, etc., se puede escribir $w a$, we, etc. El caso de las letras $c, q u, k$ que en español representan al mismo fonema (sonido diferenciador), en las lenguas indígenas puede ser escrito simplemente con la letra $k$. En lugar de tres signos, los niños pueden aprender uno solo. Pero más que el número de signos, se trata de que la escritura de la lengua debe tener un sistema sencillo y, en la medida de lo posible, ser regular. Cuántas lágrimas de los niños y niñas se evitarían en el mundo si el sistema de escritura de su lengua fuera regular; es decir, fonológica; pues disminuirían ostensiblemente las llamadas "faltas de ortografía". Las faltas de ortografía no se deben a la falta de inteligencia de los niños sino al desfase entre el habla y la escritura. El habla es dinámica, cambia constantemente, en tanto que la escritura ha quedado anquilosada, sujeta a los caprichos de los "académicos de la lengua". Evidentemente que la felicidad de los niños, al no tener tanta complejidad inútil en la escritura, disminuiría el ego de los gramáticos y de muchos "académicos". ¿Qué es preferible: que los niños disfruten de escribir su lengua con una escritura fonológica sencilla, o que los académicos se sientan importantes tratando de explicar el sin número de excepciones a la regla ortográfica? Cambiar las reglas ortográficas es algo que no podemos los educadores porque esto es ir contra el imaginario del prestigio de la ortografía presente en la mayor parte de la sociedad. Pero hay algo que todo educador sí podemos hacer, y es el dar más puntaje a la capacidad de expresión oral y escrita de los niños que a las faltas de ortografía. Lo uno es creación y lo otro solo es memorismo.

Por cuestiones de convivencia humana, es necesario organizar códigos estandarizados para escribir las lenguas y así poder entendernos, pero eso no justifica que se mantengan reglas ortográficas inútiles a la luz de la lingüística actual. El problema es que muchos "académicos" son gramáticos pero no lingüistas.

Con estos antecedentes, a continuación se señala el estado actual del establecimiento de alfabetos en las lenguas indígenas del Ecuador. En cada lengua se señalará, además, algunos datos 
sobre el estado de su estudio que podrían ser de interés para conocerlas mejor y así amar nuestro patrimonio nacional.

1) A'ingae: Esta lengua pertenece al pueblo $A^{\prime} i$ (Cofán). Éste se encuentra ubicado en los ríos Aguarico y San Miguel, en la provincia de Sucumbíos. Las comunidades donde está la población hablante de a'ingae son: Dureno, Duvuno, Sinangüe, Bermejo, Sábalo, Chandia $\mathrm{Na}$ 'en, Bavurue, Alto Bermejo, entre otras. El número de hablantes, según la profesora Enma Chica, dato proporcionado en octubre de 2006, es de cerca de mil personas en el Ecuador. Hay también hablantes de esta lengua en Colombia.

El Instituto Lingüístico de Verano (ILV) de la Universidad de Oklahoma, de los Estados Unidos, a través de sus misioneros, publicó un vocabulario del a’ingae con unas páginas introductorias de notas gramaticales.

En cuanto a la escritura, el ILV elaboró un alfabeto llamado por sus investigadores "alfabeto práctico". Lo llaman práctico por ser muy apegado al alfabeto del español.

Para el caso de las consonantes oclusivas aspiradas optaron por representarlas con la escritura duplicada de las correspondientes oclusivas simples. Los fonemas aspirados: /ph/, /chh/, /th/, /tsh/, /kh/; se representaron por las grafías pp, $\mathrm{chh}, \mathrm{tt}, \mathrm{tss}, \mathrm{cc}-\mathrm{qqu}$, respectivamente. El fonema vocálico central alto que en lingüística se representa con el signo $/ \dot{+} /$ fue representado con el grafema $<0>$.

Según los estudios del ILV la lengua a'ingae tenía que representarse de la siguiente manera: cuatro vocales orales, cuatro vocales nasales y veintisiete consonantes: $\mathrm{a}, \mathrm{an}, \mathrm{b}, \mathrm{c}, \mathrm{cc}, \mathrm{ch}, \mathrm{chh}$, d, dy, e, en, f, g, i, in, j, m, n, ñ, o, on, p, pp, qu, qqu, s, sh, t, tt, ts, tss, u, un, v, y, z, . (37 letras). En la propuesta del ILV las vocales nasales se representaban con un signo doble: el de la vocal oral junto con una n. Así por ejemplo: an, en, in, etc.

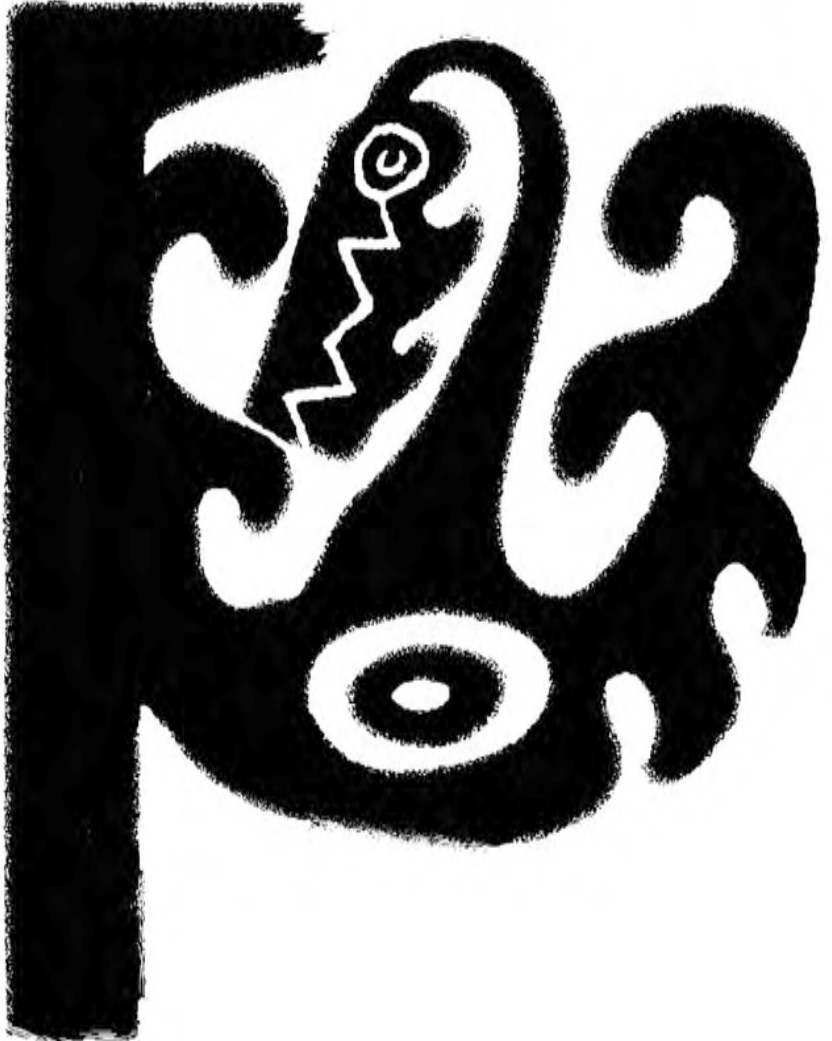

Al haber existido una tradición de más o menos unos veinticinco años de esta escri-

tura y al haber sido impresa la biblia en aingae con esta escritura, nadie había hecho el intento de cambiarla.

Luego de que la Dirección Nacional de Educación Intercultural Bilingüe DINEIB a través del Programa Universitario del Sistema de Educación Intercultural Bilingüe para la paz PUSEIB-paz, organizó algunos cursos de lingüística a fines del año 2006 e inicio de 2007, los educadores acordaron en una reunión en Lago Agrio del 15 al 19 de enero de 2007, realizar algunos cambios a la escritura del ILV. Entre los cambios más importantes era el de reemplazar las letras c, qu por la letra k. También se representaba la aspiración en las consonantes por la letra h. Así pues, en lugar de escribir con letras dobles como pp, tt, etc, se escribiría ph, th. el alfabeto con los siguientes grafías: $a, b, c h, c h h, d$, dy, e, f, g, i, j , k, kh, m, n, ñ, p, ph, s, sh, t, th, ts, 
tsh, u, û, v, y, z, ‘. (30 letras). Se eliminaría las vocales nasales por considerar que estas vocales ya se habían segmentalizado y se podía representar con la letra n.

Esta escritura, tampoco parece ser la definitiva, pues algunos líderes estiman conveniente hacer una nueva reunión para decidir los cambios. También parece interesante que se realicen estudios y reuniones binacionales con Colombia para que puedan adoptar una escritura estandarizada para los dos países. Además es necesario que exista una identidad común para todos sus hablantes, más allá de las fronteras nacionales.

Los educadores $A^{\prime} i$, están elaborando un vocabulario infantil ilustrado por ellos mismos.

2) Awapit: Esta lengua es hablada por la población awa. Sus habitantes la conocen con el nombre de awapit [awabit]. En Ecuador, el número de hablantes es de unos tres mil quinientos, que se encuentran en las fronteras de Carchi y Esmeraldas y en la parte noroccidental de Imbabura. Se hallan conformando diecisiete centros, de los cuales un sesenta por ciento son de habla awapit, el resto han perdido la lengua y se han castellanizado. Los centros donde el idioma awapit tiene gran vitalidad son Mataje y San Marcos. En zonas como La Guaña y El Baboso se ha perdido el idioma.

Otro grupo de unos diez mil hablantes se encuentra en Colombia, en la Costa del Departamento de Nariño y en el Putumayo en la parte amazónica, junto al poblado de Villagarzón. A la parte amazónica de Colombia han emigrado los awas hace varias décadas.

En relación a las investigaciones lingüísticas cabe mencionar que el señor Lee Henriksen, del ILV de Colombia, ha investigado por un espacio de treinta años, a partir de la década del sesenta, principalmente un dialecto del awapit de Colombia. En la década de los ochenta, la Confederación de Nacionalidades Indígenas del Ecuador (CONAIE) con la Unidad Técnica del Plan Awa UTEPA y representantes del Instituto Caro y
Cuervo, Universidad de Nariño e Instituto Colombiano de Antropología comenzaron estudios del idioma awapit. Por parte de la CONAIE trabajó Luis Montaluisa, quien en las dos reuniones binacionales de 1987 presentó una propuesta de alfabeto para unificar los dialectos ecuatorianos y colombianos. El licenciado Enrique Contreras ha realizado estudios sobre el awapit en el lado ecuatoriano.

La mayor divergencia en la escritura del awapit entre el Dr. Lee Henriksen y el de Luis Montaluisa fue la inclusión o no de la consonante $\mathrm{r}$. Esto presentaba una seria dificultad para la teoría lingüística, particularmente para la fonología. El Dr. Henriksen planteaba no incluir esta letra por cuanto era sólo una variante del fone$\mathrm{ma} / \mathrm{t} /$ en posición intervocálica.

Aplicando el concepto común de los pares mínimos, el Dr. Henriksen tenía razón. Sin embargo, Luis Montaluisa, desde 1987, consideró que dado el nivel de evolución del sonido [r] se podía considerar que se trataba de un fonema en un avanzado estado de formación. A esta situación la denominó un cuasi-fonema: por ello, era conveniente introducirlo en la escritura. Finalmente en 1996, los awa tanto de Colombia como del Ecuador, en una reunión binacional en Peguche, provincia de Imbabura, con auspicio de la Cancillería, la GTZ y otros organismos de Ecuador y de Colombia, consideraron que la propuesta de Luis Montaluisa de incluir la $\mathrm{r}$ en el alfabeto era aceptable. Otro punto discordante era la presencia de consonantes geminadas según el criterio del Dr. Henriksen. Luis Montaluisa consideró que simplemente se trataba del encuentro entre un fonema de final de una palabra y un fonema similar de comienzo de otra palabra. Eran pues sólo palabras compuestas con una frontera consonántica común. Por ejemplo, en la palabra ippa (trueno) el Dr. Henriksen consideraban que había el sonido geminado /pp/ como un fonema geminado. Pero si se analiza con cuidado, se encuentra que esta palabra está compuesta de dos palabras que son: ip (sonido) y pa (sol). Por lo tanto la primera $\mathrm{p}$ corresponde al sonido final de 
la ip y la segunda $\mathrm{p}$ corresponde al primer sonido de la segunda palabra.

De esta manera, el alfabeto del awapit que se está utilizando es el siguiente: a, â, ch, e, i, î, ih, $\dot{i}$, î, th, j, k, l, m, n, ñ, p, s, sh, t, u, û, uh, w, y, ${ }^{c}$. (26 letras).

Se debe aclarar que, al parecer, el fonema e es de reciente formación y sólo se halla en el morfema topicalizador $\{$-ne $\}$, que seguramente proviene del morfema $\{$-nain $\}$, punto que debe dilucidarse con estudios posteriores.

Luis Montaluisa ha recopilado un corpus de unas dos mil palabras, documento que todavía permanece inédito. Apenas se ha iniciado un estudio de la morfología del awapit. Se ha detectado que el verbo tiene una gran riqueza en las "formas aspectuales". En el campo de la semántica se han realizado algunos avances, particularmente en la constatación de ser un idioma conformado por un léxico con abundantes palabras compuestas y basadas en monosílabos.

Los awa han publicado con la DINEIB algunos mitos y cuentos en lengua awapit.

3) Cha'fiki (hasta hace poco conocida como cha'palaa). Es la lengua del pueblo chachi; conocidos por algunos historiadores como cayapas, debido a que una parte de la población actualmente está asentada en el río del mismo nombre. El número de hablantes es de unos ocho mil aproximadamente. Se encuentran en la zona del río Cayapas, del río Canandé y de Muisne, en Esmeraldas.

El ILV ha publicado un vocabulario con algunas notas gramaticales. El padre Vitadello ha publicado dos tomos sobre la lengua cha'palaa, el uno sobre aspectos morfosemánticos y el otro sobre mitología y narración. Es importante destacar que un indígena chachi, José Francisco Añapa, ha elaborado este año un pequeño, pero interesante, estudio sobre su lengua denominado "Módulo para el Área del Idioma Cha'palaa".

El alfabeto chapalaa que se está comenzando a usar es el siguiente: a, aa, b, ch, d, dy, e, ee, f, g, i, ii, j, k, l, ll, m, n, ñ, p, r, s, sh, t, ts, ty, u, uu, v, $y,$. (30 letras).

Anteriormente, el ILV y otros autores habían escrito esta lengua con las letras c, qu, la h muda, etc.

4) Epera Pedede (pronunciado êpera pedee): Esta lengua pertenece al pueblo êpera (embe$r a)$, grupo reducido en el Ecuador. En Colombia, está conformado por unas treinta mil personas. En nuestro país los hablantes de esta lengua se encuentran en la provincia de Esmeraldas, frente a la población negra de Borbón, en la confluencia del río Santiago con el Cayapas. Su número se estima en alrededor de sesenta personas.

Para el dialecto ecuatoriano sólo existe un estudio de la fonología del êpera pedede realizado para la CONAIE por Catalina Álvarez. De acuerdo al estudio el alfabeto podría ser el siguiente: a, â, aa, b, ch, d, e, ê, ee, i, î, ii, i, î, ii, j, k, kh, m, n, o, ô, oo, p, ph, r, s, t, th, u, û, uu, w, y. (32 letras).

Falta realizar un estudio comparativo con los otros dialectos colombianos para intentar establecer un sistema de escritura unificado para todos los dialectos del êpera pedede.

A partir del 2006 parece que hay algún intento de analizar esta lengua por otros estudiosos.

5) Kayapi (Záparo): El pueblo Kayapi se encuentra ubicado en el Curaray, en Llanchama Cocha al norte de la provincia de Pastaza y en Balsaura al noreste de Pastaza; la lengua que hablan se la conoce como Záparo. Hay muy pocas familias que lo hablan. Se dice que antiguamente el pueblo Záparo era muy numeroso, pero actualmente apenas quedan algunos pocos representantes de esta cultura, a lo largo del río Curaray.

Según Gaetano Osculati, en su libro Esplorazione delle regioni equatoriali lungo il Napo ed il fiume delle Amazzoni (Milano, 1850), habían unos 20.000 hablantes. A fines del siglo XVII se estimaba en 98.500 parlantes. 
Los estudios más importantes sobre esta lengua son:

Rivet, Paul. 1930. Contribution a l'etude des tribus indiennes de l'Orient Equatorien, "Bulletin de la Société des Américanistes de Belgique, pp. 7-8.

Sargeant, Mary. 1950. Vocabulario Zaparo: Estudios acerca de las lenguas huaorani (auca), shimigae y zapara. Publicaciones Científicas del Ministerio de Educación del Ecuador, pp. 43-48.

El padre León, de la misión dominicana, a comienzos del siglo XX, realizó pequeñas recopilaciones sobre este idioma.

Luis Montaluisa realizó una recopilación de vocabulario del habla zápara de Llanchamacocha, Curaray en 1996. Además el propuso que las abuelas enseñen a los niños el idioma. Este proceso parece que se está realizando con bastante interés por los sapara. La historia dirá si la lengua sobrevive o desaparece para siempre.

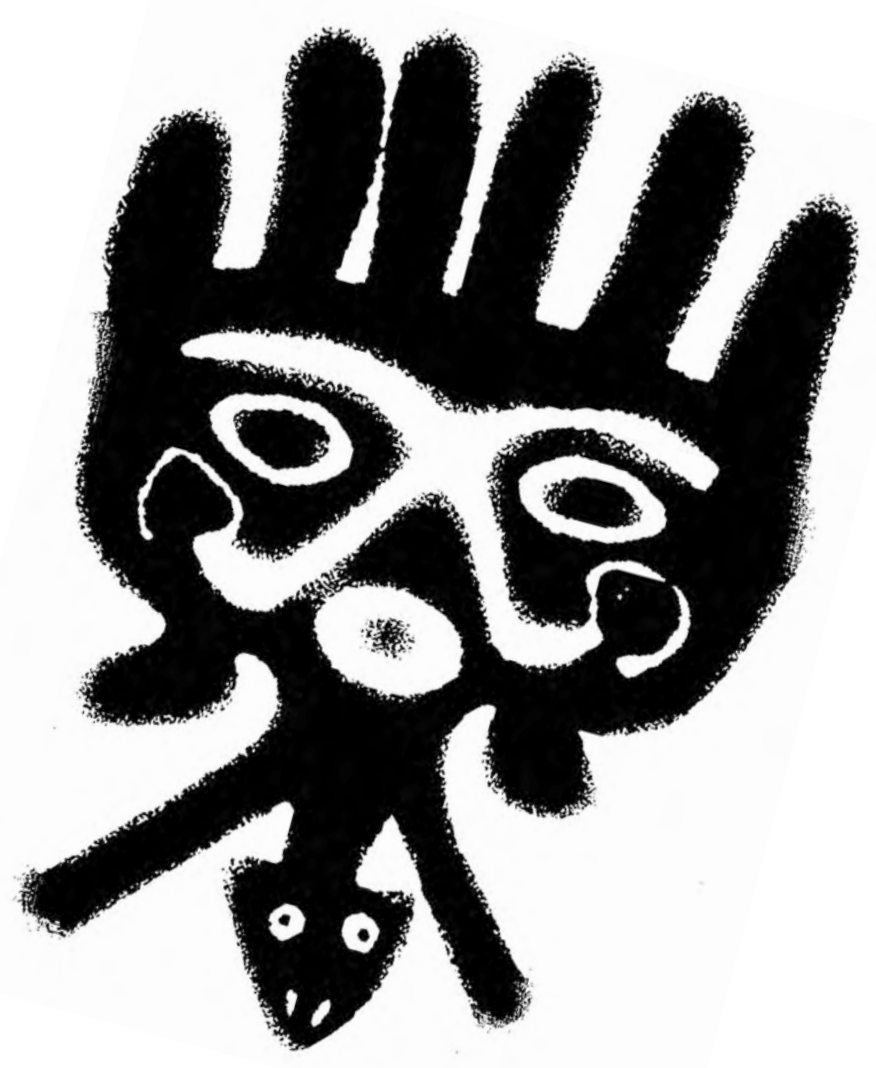

La recopilación del corpus lingüístico más importante la hizo Louisa Stark entre septiembre de 1971 y agosto de 1975 . Este trabajo poco conocido en los medios lingüísticos comprende un vocabulario de unas 500 palabras.

El alfabeto empleado por Louisa Stark es el siguiente: a, ch, i, j, k, m, n, p, r, s, sh, t, ts, u, w, y, $\mathrm{zh}^{*}$, ‘. (17 letras). Existe la posibilidad de que existan vocales nasales, lo cual deberá ser confirmado con posteriores investigaciones. La zh sólo se encuentra en préstamos del quichua.

Existen intentos aislados por tratar de rescatar la lengua zápara, en la actualidad sólo es hablada por personas mayores de 65 años, que son quienes enseñan a los niños de la comunidad. El temor existente es que sólo aprendan el vocabulario pero no la conversación. Parece inminente la muerte de esta lengua en el dialecto ecuatoriano.

En estos últimos años, el Sr. Jorge Trujillo por su lado y el Sr. Carlos Pallares por el suyo han recopilado vocabulario, mitos y tradiciones de la cultura zápara.

Hace tres años la UNESCO declaró a la lengua y cultura zápara como patrimonio intangible de la humanidad.

Catalina Álvarez realizó, en el 2001, un estudio de esta lengua para apoyar la elaboración de materiales educativos en la Dirección de Educación Zápara.

Cristóbal Quishpe, del PUSEIB-paz DINEIB, también ha realizado estudios de esta lengua y últimamente la Dra. Ruth Moya está realizando un estudio gramatical de la lengua.

Actualmente, luego de un proceso organizativo de algunos años, es la Organización de la Nacionalidad Zápara del Ecuador -NAZAE- la que representa a esta nacionalidad. Sin embargo, falta consolidar el proceso organizativo de todas las comunidades sáparas (Los líderes de esta nacionalidad prefieren escribir el nombre de la nacionalidad con la letra s en lugar de la z).

Están en proceso definir el alfabeto y elaborar materiales educativos para con el apoyo de algunos proyectos como "Educación Intercultural 
Bilingüe para la Amazonía EIBAMAZ", con financiamiento de Finlandia y el Proyecto Sasico, con Financiamiento de Cataluña.

Los educadores sapara están realizando un vocabulario infantil ilustrado de su lengua.

6) Pạikoka: o Baikoka. Es la lengua de los pueblos Siona y Secoya. Desde el punto de vista lingüístico estas dos hablas son dialectos de una misma lengua. Pero desde el punto de vista de la identidad, cada pueblo se considera una nacionalidad, basado en aspectos sociohistóricos. El número de secoya-hablantes es de doscientas cincuenta personas y el de siona-hablantes es de doscientas a ciento cincuenta personas.

Se encuentran ubicados en los ríos Aguarico y Cuyabeno en la provincia de Sucumbíos. Los centros Siona son el de Piaña (Campo Eno) y Puerto Bolívar y los centros Secoya son el de San Pablo de Cantetsiaya y el de Siecoya (sewaya).

La lengua Paikoka tuvo el dialecto conocido con el nombre de tetete que en lengua A'ingae significa salvaje; ésta perteneció a un grupo errante asentado cerca del río Putumayo. Parece que este dialecto está extinto a partir de la década de los ochenta. Posiblemente sus hablantes se quichuizaron.

Existen hablantes de Paicoca en Colombia y Perú. En Colombia hablan el dialecto Siona y en Perú el Secoya. Además, esta lengua tiene estrecha relación con todas las de la gran familia TUCANO que se encuentran en Colombia, Perú y Brasil. El Paicoca forma parte del grupo de TUCANO OCCIDENTAL.

El ILV del Ecuador ha publicado un vocabulario y una gramática del dialecto secoya. En Ecuador no se han realizado estudios del dialecto siona. En Colombia, el ILV de ese país realizó estudios del siona.

Luis Montaluisa, por su actividad docente, ha realizado una comparación de los dialectos siona y secoya encontrando que la diferencia fundamental radica en que los sionas habían sonoriza- do el fonema oclusivo bilabial sordo /p/. Ejemplo [pãikoka bãikoka] (nombre del idioma que significa idioma de la gente).

También se encontró que los secoyas han nasalizado en algunos contextos el fonema oclusivo dental sonoro /d/. Ejemplo [domio nomio] 'mujer'. Lejos de esto existe una diferencia a nivel de léxico en un porcentaje estimado en un cinco por ciento. Estas diferencias en la práctica podrían ser superadas al estandarizar los dos dialectos. Sin embargo, existe una reacción de los sionas debido a que consideran que las instituciones, comenzando por el propio ILV, han apoyado al desarrollo de los secoyas, y no han prestado atención al pueblo siona.

Este problema podría ser superado con la inclusión de las particularidades lexicales del siona en el diccionario unificado. El pedido de los sionas de escribir con $\mathrm{b}$ al fonema / $\mathrm{p} /$ no procedería, por ser únicamente una variante dialectal.

El alfabeto que estableció el ILV para esta lengua tiene seis vocales orales, seis vocales nasales y quince consonantes: a, $\underline{\mathrm{a}}, \mathrm{c}, \mathrm{d}, \mathrm{e}, \underline{\mathrm{e}}, \ddot{\mathrm{e}}, \underline{\ddot{e}}, \mathrm{hu}, \mathrm{i}, \underline{\mathrm{i}}, \mathrm{j}$, m, n, $\tilde{\mathrm{n}}, \mathrm{o}, \underline{\mathrm{o}}, \mathrm{p}, \mathrm{qu}, \mathrm{r}, \mathrm{s}, \mathrm{t}, \mathrm{ts}, \mathrm{u}, \underline{\mathrm{u}}, \mathrm{y}$, . . (27 letras).

En enero de 2007 los Sionas y Secoyas decidieron cambiar la c, qu por la letra $\mathrm{k}$ y la hu por la letra w. En este caso el alfabeto quedaría con 26 letras. Aclarando que los secoyas usan la letra $\mathrm{p}$, en tanto que los siona prefieren usar la letra $b$. Otras diferencias entre las dos hablas están en el uso de la ch, la ts, la s, la d, n, etc.

Cada idioma indígena tiene un aporte importante a la ciencia lingüística universal. Por ejemplo, el Paikoka es el único idioma ecuatoriano que indica género en el verbo. Así:

$$
\begin{array}{ll}
<\text { saiji }> & \text { 'va' (él) } \\
<\text { saico } & \text { 'va' (ella) }
\end{array}
$$

7) Quichua o Runa Shimi: Los pueblos quichua hablantes se ubican en las provincias del callejón interandino y en la mayoría del oriente: Imbabura, Pichincha, Bolívar, Cotopaxi, Tungurahua, Chimborazo, Cañar, Azuay, Loja, Napo, Sucumbíos, Orellana, Pastaza y Zamora Chinchipe. 
Hay también grupos de migrantes indígenas en algunas provincias de la costa que mantienen su lealtad a la lengua quichua, y la siguen hablando.

Fuera del Ecuador hay quichua-hablantes en Perú, Bolivia, Argentina, Colombia, Brasil, Chile y posiblemente, en Paraguay. Entre todos los países, el número total de quichua-hablantes se estima en ocho millones.

Sobre este idioma se ha realizado varias publicaciones de diccionarios y gramáticas desde la época de la Colonia.

En cuanto a la escritura, a partir de Paul Rivet y Jijón y Caamaño que desde las primeras décadas del presente siglo introdujeron la $\mathrm{k}$ y la w para la escritura de idiomas indígenas, existe la tendencia para escribir con este sistema internacional.

El proceso de establecimiento de la escritura de una lengua, es complejo. La complejidad no se debe tanto al estudio lingüístico, sino al hecho de que la escritura es una construcción psicosocial. Intervienen factores de poder político, religioso.

La Pontificia Universidad Católica del Ecuador, con sus profesores Dra. Consuelo Yánez y Lic. Fausto Jara, elaboró un método para la enseñanza del quichua en el que se empleaba esta escritura. Posteriormente, en 1980, en una reunión de líderes indígenas, en el Campamento Nueva Vida, se acordó no utilizar la k y la w. Se aprobó el siguiente alfabeto: a, b, c, ch, d, f, g, h, i, j, l, ll, m, n, ñ, p, qu, r, s, sh, t, ts, u, y, z, zh. (26 letras). En las semanas siguientes, personal de la Universidad Católica con los promotores nacionales para la alfabetización designados en este reunión, redujeron este alfabeto al siguiente: a, c, ch, hu, i, j, l, ll, m, n, ñ, p, qu, r, s, sh, t, ts, $u, y, z$. (21 letras). Escritura que se mantiene hasta hoy.
Hace unos siete años en una reunión de algunos educadores de la DINEIB resolvieron volver a emplear las letras k, w. Luis Montaluisa, desde 1985 viene proponiendo que se estandarice la escritura a nivel de todos los dialectos de todos los países de América donde se lo habla. Según Montaluisa, éste podría ser el alfabeto a ser usado para la escritura del quichua: $a, c h, \operatorname{tr}^{*}, i, h(j)$, k, l, ll, m, n, ñ, p, q, r, s, sh, t, u, w, y. (20 letras). Para el caso del quichua ecuatoriano esto significa la reintroducción del fonema postvelar /q/. Este fonema en realidad existe en los dialectos del quichua de la Sierra Central (Chimborazo, Bolívar), pero no había sido registrado por los estudiosos del quichua. Igualmente, habría que introducir el fonema /tr/, si es que los estudios indican su pertinencia. De los dialectos del sur del Perú y de Bolivia habría que reconsiderar los fonemas oclusivos aspirados y glotalizados: $\mathrm{ph}$, th, chh, kh, qh, p', t', ch', k', q'. Estas grafías se emplearían solo para las palabras que comparten el kichwa y el aymara.

El sonido [1] de los dialectos ecuatorianos, si los estudios así lo sugieren, tend ría que desaparecer, pues palabras como [lulun] en Cañar la pronuncian [ruru]. Además existen pocas palabras con este sonido.

Los sonidos [ts], [zh], [z], se encuentran únicamente en palabras de origen indígenas prequichuas. El sonido [ts] presente en algunas palabras de Imbabura como en [tsawar] 'penco, cabuyo', son variaciones del fonema /ch/. Pero en el caso de la Amazonía ecuatoriana hay varias palabras con ts, posiblemente como un sustrato de lenguas indígenas anteriores al quichua. En este caso habría que mantener en la escritura la grafía ts. Algo similar sucede con la presencia del sonido [ts] en el dialecto de Ancash en el Perú.

Hoy el alfabeto que se está empleando para escribir el quichua en el Ecuador es: a, ch, h(j), I, k, l, ll, m, n, ñ, p, r, s, sh, t, u, w, y. (18 letras). En la Amazonía prefieren escribir con j en lugar de la h.

Hay varios diccionarios y gramáticas sobre el quichua, desde 1560. En cuanto a la concepción y estructura. Hay un diccionario ilustrado 
denominado Caimi Nucanchic Shimiyuc-panca, elaborado en 1982 en el Centro de Investigaciones para la Educación Indígena CIEI de la PUCE en el marco de un convenio con el Ministerio de Educación. En este diccionario, dirigido por Luis Montaluisa, por primera vez, las definiciones, ejemplos, explicaciones y toda información se hizo en quichua. Se incluyó las variantes fonéticas de cada palabra, los sinónimos, etc. En la investigación se consideró todas las variantes de todas las provincias del Ecuador, donde se habla esta lengua.

Los educadores indígenas están elaborando vocabularios infantiles ilustrados de esta lengua.

Los alumnos indígenas de la Universidad de Cuenca en convenio con la DINEIB han realizado investigaciones sobre la lengua y la cultura shuar, dirigido por el Dr. Alejandro Mendoza.

Fabián Potosí, José Caiza y otros indígenas han escrito vocabularios sobre el quichua. En la Amazonía han estudiado varias personas: Mariano Cerda, el padre Camilo Mugica. El padre José Miguel Goldaraz escribió algunos cinco tomos sobre mitología y tradiciones quichuas. Uno de ellos totalmente en quichua. Estos han sido publicados por CICAME coordinados por el padre Miguel Angel Cabodevilla.

La Dra. Consuelo Yánez ha elaborado un léxico en dos tomos con más de quince mil palabras, publicado por Abya-Yala. También ella ha reeditado el método para la enseñanza del quichua como segunda lengua.

Varias universidades tienen programas de educación vinculadas con hablantes del quichua: Universidad de Cuenca, Universidad Politécnica Salesiana. Otras han tenido varias promociones para estudiantes indígenas de esta lengua: Universidad Técnica Particular de Loja UTPL, Universidad de Bolívar, etc.

8) Shuar-Achuar-Shiwiar Chicham: Los historiadores tradicionales lo conocen también como lengua "jívara” o “jíbara” El número de hablantes de esta lengua en Ecuador es de unos cincuenta mil. En el Perú existe un nú- mero similar de personas que lo hablan. En el país, los parlantes están ubicados en Zamora Chinchipe, Morona Santiago y Pastaza. Recientemente hay grupos en Napo, Sucumbíos y también en algunas provincias de la Costa.

Junto con el shuar-chicham ('lengua de la persona'), están el achuar y el shiwiar. El dialecto achuar se habla en las zonas fronterizas con Perú de las provincias de Morona Santiago y Pastaza.

Existe una importante variación lexical entre los dialectos shuar y el dialecto achuar. Por ejemplo, el número tres en shuar se dice /menaint/, en tanto que en achuar se dice /kampata/; el cuatro en shuar es /aintiuk/, en achuar es /chinduk-chin$d u k l$, etc.

Cabe destacar que dentro del dialecto shuar existen varios subdialectos entre ellos está el habla del sur (Bomboiza, Zamora, etc.) y la del norte (Sucúa, Chiguaza, etc.). También existe otro subdialecto, en la provincia de Pastaza conocido como shiwiar.

La dificultad mayor está en la unificación entre los dialectos shuar, achuar y shiwiar, por la importante presencia de variaciones lexicales, agravada con los problemas extralingüísticos como son los de carácter socio-organizativo.

Es importante la unificación con los dialectos shuar-achuar del Perú. Allí los han bautizado con los nombres de Huambisa, Aguaruna (aguajun), achual, etc., pero son de la misma familia shuar-achuar.

El proceso de Paz Ecuador-Perú debe contribuir a la estandarización de la escritura a nivel supranacional para idiomas como el shuarachuar, paicoca, quichua, etc. Igualmente con Colombia el ejemplo seguido por el awapit debería hacerse con los idiomas a'ingae, epera pedede y paicoca.

El ILV empleó el siguiente alfabeto: a, ch, e, i, j, k, m, n, p, r, s, sh, t, ts, u, w, y. (17 letras). Los misioneros salesianos añadieron las vocales nasales y unas vocales que las llamaron susurradas.

En el shuar se emplea el siguiente alfabeto: cuatro vocales orales, cuatro vocales nasales y 
cuatro vocales largas y trece consonantes: a, a, aa, ch, e, e e ee, i, $\underline{\mathrm{i}}, \mathrm{ii}, \mathrm{j}, \mathrm{k}, \mathrm{m}, \mathrm{n}, \mathrm{p}, \mathrm{r}, \mathrm{s}, \mathrm{sh}, \mathrm{t}, \mathrm{ts}, \mathrm{u}, \underline{\mathrm{u}}, \mathrm{uu}$, $\mathrm{w}, \mathrm{y}$. (25 letras). Al esclarecimiento de la fonología de esta lengua contribuyó la tesis realizada por Santiago Utitiaj Paati de la Universidad Politécnica Salesiana. Algunos hablantes sugieren que se debe decir que el shuar chicham sólo tiene cuatro vocales fundamentales más un fonema de nasalización y otro de alargamiento de las mismas cuatro vocales básicas.

En el achuar se está empleando el siguiente alfabeto: a, aa, ch, e, ee, i, ii, j, k, m, n, p, r, s, sh, t, ts, $u, u u, w, y$.

Actualmente están elaborando un vocabulario infantil ilustrado en las variantes: achuar, shiwiar y shuar..

9) Tsafiqui: Es la lengua que habla el pueblo Tsa'chi conocido por los hispano-hablantes como "colorados". Este grupo se halla ubicado en la zona de Santo Domingo de los Colorados en la provincia de Pichincha. El número de hablantes es de unos dos mil, los que se encuentran esparcidos en ocho comunidades. Ellas son: Bua, Chiguilpe, Cóngoma, Naranjos, Peripa, Poste, Otongo y Tahuaza.

Existen variaciones dialectales entre las hablas de Cóngoma y las demás. Por ejemplo, perro en Cóngoma se dice [shushu], mientras en otros lugares se dice [susu], la lluvia en Cóngoma es [shua], en otros lugares se dice [sua]. En la actualidad se ha preferido mantener en la escritura la sh para las palabras donde existe su presencia en algunas hablas y la s para las palabras que utilizan este sonido en todas las hablas.

El alfabeto tsa'fiki que se usa hoy es el siguiente: a, ch, d, e, f, g, i, j, k, l, m, n, ñ, o, p, r, s, sh, t, ts, u, w, y. (23 letras).

10) Waotededo (se pronuncia wao terero o wao tiriro): Los hablantes de esta lengua están ubicados en los ríos Yasuní, Cononaco, Nushiño y Curaray de las provincias de Napo y
Pastaza. Tiene aproximadamente unos mil trescientos parlantes.

Existen variaciones entre las hablas del Yasuní, Cononaco, etc., sin embargo no parece difícil su unificación.

El alfabeto propuesto y usado inicialmente por el ILV fue el siguiente: a, ä, b, c, d, e, ë, ae, aë, g, i, ï, o, ö, p, qu, t, w, y. (19 letras).

Luis Montaluisa propuso el siguiente alfabeto: a, b, d, e, g, i, k, m, n, ñ, ng, o, p, t, w, y. (16 letras). Se suprimían las vocales nasales porque en las últimas décadas ha existido un proceso de segmentalización de la nasalidad suprasegmental de las vocales. Al segmentalizarse las nasales se las escribe con las grafías $m, n, \tilde{n}, n g$, por lo tanto, ya no hace falta representar las vocales nasales. Ejemplo: <onko $>$ 'casa' no es necesario escribir $<\ddot{o} k o\rangle$.

El sonido [ae] hay que estudiarlo para ver la necesidad o no de incluir en el alfabeto. El ILV ha admitido la inclusión de las grafías $\mathrm{m}, \mathrm{n}, \tilde{\mathrm{n}}, \mathrm{ng}$, pero mantiene la grafía ae.

La dirección de educación wao con la DINEIB están organizando un programa para producir materiales educativos en esta lengua, pero para ello es necesario con la discusión de la escritura. Esto lo realizarán del 5 al 8 de marzo de 2007 en el Puyo.

11) Andoa. Se desconoce el nombre propio de esta lengua. Sus hablantes están en la provincia de Pastaza. Hay algunos miembros de esta nacionalidad que viven el actual territorio peruano, siguiendo el curso del río Pastaza. Todavía no se ha estandarizado la escritura del idioma.

Un dato común de los nombres de los idiomas indígenas ecuatorianos es que unen la noción de gente con lengua. Traduciendo al español, los nombres de las lenguas indígenas, todas significan lengua del hombre (persona).:

- a'ingae (a'I = hombre; ingae = lengua)

$=$ lengua del hombre 
- $\quad$ awapit $($ awa $=$ hombre; pit =lengua $)$ = lengua del hombre

- cha'fiki (chachi =hombre; fiki =lengua) $=$ lengua del hombre

- $\quad$ epera pedede (êpera = hombre; pedede =lengua $)$ $=$ lengua del hombre

- $\quad$ paicoca $(p \underline{a} \mathbf{i}=$ hombre; $\operatorname{coca}=$ lengua $)$ = lengua del hombre

- runa shimi $($ runa = hombre; $\operatorname{shimj}=$ lengua $)$ = lengua del hombre

- shuar-chicham $($ shuar =humano; chicham =lengua $)$ = lengua humana

- $\quad$ tsa'fiqui (tsachi =hombre; fiqui =lengua) $=$ lengua del hombre

- wao-tededo (wao = hombre; tededo =lengua) $=$ lengua del hombre.

La riqueza de las lenguas indígenas está en el vocabulario relacionado con la flora y fauna. Es- tos nombres junto con los topónimos, antropónimos constituyen la identidad milenaria del Ecuador. Hay que evitar que se cambien los nombres propios de las lenguas indígenas por nombres de los invasores. La historia milenaria se puede reconstruir tanto a través de los restos arqueológicos como de los topónimos, fitónimos, antropónimos, ancestrales, etc.

Para asegurar el desarrollo sustentable del Ecuador hay que redescubrir la historia milenaria y realizar una adecuada administración de los recursos naturales, mediante un Plan de Estado a largo plazo. El agua, la flora y la fauna son los mayores recursos naturales del Ecuador. El estudio de las lenguas indígenas hay que colocarla en este contexto. Su estudio, puede contribuir a la comprensión del ser humano, a la construcción de la paz y la interculturalidad.

1 Máster en Educomunicación, Doctora en Jurisprudencia, Licenciada en Ciencias Políticas y Sociales, Licenciada en Lingüística Andina y Educación Bilingüe; Docente UPS.

2 Lingüista y Doctor en Ciencias de la Educación por la Pontificia Universidad Católica del Ecuador PUCE, Máster en Seguridad y Desarrollo por el Instituto de Altos Estudios Nacionales IAEN, Máster en Recursos Naturales por la Universidad Politécnica Salesiana UPS, PhD Honoris Causa por las Universidades del Consejo Iberoamericano de Educación 2005; Técnico DINEIB, Docente UPS.

3 Diglosia es la actitud de una sociedad multilingüe, en la cual una lengua es considerada superior y las otras son consideradas inferiores. El español se afianzó como lengua superior y las lenguas indígenas como lenguas inferiores. De la misma manera, la forma de vida extranjerizante se convirtió en el modelo de vida para los ecuatorianos. Para la ciencia lingüística todas las lenguas tienen el mismo valor. Todas sirven para comunicarse. Pese a estos avances de las ciencias, en el imaginario de la mayor parte de los ecuatorianos está presente la idea de que las lenguas y culturas indígenas son inferiores. Corresponde a los comunicadores y a los educadores destruir estos falsos mitos. Para ello es necesario conocer lo más que se pueda de las lenguas y culturas indígenas que han logrado sobrevivir a pesar de la ideología criolla impuesta. Con razón el historiador de la economía ecuatoriana José Corsino Cardenas, considera a los historiadores tradicionales del Ecuador como "encomenderos disfrazados de historiadores". 